\title{
A preliminary assessment of a combination of rhodiola and saffron in the management of mild-moderate depression
}

This article was published in the following Dove Press journal:

Neuropsychiatric Disease and Treatment

\author{
Marie Bangratz' \\ Samira Ait Abdellah' \\ Aurélie Berlin' \\ Claude Blondeau' \\ Angèle Guilbot' \\ Michel Dubourdeaux' \\ Patrick Lemoine ${ }^{2}$ \\ 'Groupe PiLeJe, Paris, France; \\ ${ }^{2}$ Clinique Lyon Lumière, Meyzieu, \\ France
}

Correspondence: Claude Blondeau Groupe PiLeJe, 37 Quai de Grenelle, Paris 75015 , France

Tel +33 I5 3867078

Email c.blondeau@pileje.com
Objective: The medicinal plants Rhodiola rosea L. (rhodiola, golden root) and Crocus sativus L. (saffron) have been shown separately to induce significant effects in depression. The objective of this study was to assess a fixed combination of rhodiola and saffron in mild-moderate depression.

Methods: In this observational study conducted with general practitioners (GPs), 45 adults (aged 18-85 years) suffering from mild or moderate depression (International Statistical Classification of Diseases and Related Health Problems 10th Revision definition) and reaching a score on the Hamilton Rating Scale for Depression of 8-18 were supplemented with a combination of rhodiola and saffron extracts (one tablet, $154 \mathrm{mg}$ of rhodiola and $15 \mathrm{mg}$ of saffron; recommended dose two tablets per day for 6 weeks).

Results: After 6 weeks (D42) of supplementation, Hamilton Rating Scale for Depression scores (primary outcome) decreased significantly by $58 \% \pm 28.5 \%$ (from $13.6 \pm 2.3$ at D0 to $5.6 \pm 3.8$ at D42, $P<0.0001 ; n=41)$. Score improvement was reported in $85.4 \%$ of patients. A significant drop in both Hospital Anxiety and Depression Scale anxiety and depression scores was also observed at D42, the decrease being significant from 2 weeks of supplementation. At the end of the study, both GPs and patients deemed there was a significant improvement in depression (Clinical Global Impression - improvement and Patient Global Impression of Change). Safety was excellent, and no serious adverse effects were recorded.

Conclusion: Results of this observational study performed in primary care suggest that the combination of rhodiola and saffron tested could be useful for the management of mild-moderate depression and improve depressive and anxiety symptoms. A double-blind placebo-controlled study is needed to confirm these results.

Keywords: major depressive disorder, anxiety, Rhodiola rosea, Crocus sativus

\section{Introduction}

Depression is one of the most frequent and debilitating psychiatric conditions. ${ }^{1-3}$ It has been reported that approximately one in five adults experiences at least one episode of depression in their lifetime, women being twice as likely to develop this disorder. ${ }^{1,4}$ Depression is also widely underdiagnosed and undertreated, despite the wide range of treatments available..$^{1,2,4,5}$ It is estimated that $<25 \%$ of depressive patients receive adequate treatment according to expert guidelines. ${ }^{5,6}$ In addition, conventional antidepressants are often associated with incomplete response, low compliance, low remission rates, high risk of relapse, substantial side effects, low tolerability, and premature discontinuation. ${ }^{1,6,7}$ Findings indicate that the efficacy of antidepressants varies considerably as a function of symptom severity. ${ }^{6,8}$ It has been reported that whereas antidepressants can be quite potent with more severe depression, there is little evidence 
to suggest that they produce any benefit for the majority of patients with less severe forms of depression. ${ }^{8}$ In addition, many patients with milder symptoms worry about side effects of antidepressants. ${ }^{9}$ Certain guidelines for the management of depression recommend antidepressants for moderate-severe depression and not for mild depressive episodes, because the risk:benefit ratio is extremely poor. Psychological treatment, such as cognitive behavioral therapy, is recommended, ${ }^{10-12}$ while other guidelines indicate either an antidepressant or psychotherapy (or both) in mild-moderate depression and both antidepressants and psychotherapy in severe or chronic forms of depression. ${ }^{13,14}$ Psychological treatment, such as cognitive behavioral therapy, can be an effective alternative to pharmacotherapy. ${ }^{15}$ However, since qualified therapists are rare and expensive, treatment access is quite low. ${ }^{16}$ Therefore, there is a need for alternative or complementary medicines for less severe forms of depression to provide more favorable outcomes and easier access, especially since the majority of depressive patients suffer from mild-moderate forms of depression. $^{5}$

Herbal medicines have been explored as a source for alternatives to conventional antidepressants, among which is saffron, a spice from the Middle East extracted from the flower of Crocus sativus L. Results of several preclinical studies and clinical trials have shown that saffron has beneficial effects in depression. ${ }^{17-24}$ Supplementation with saffron was found to be as effective as treatment with conventional antidepressants, such as imipramine and fluoxetine, in randomized double-blind clinical trials. ${ }^{17,19,23}$ Another plant with interesting properties is Rhodiola rosea L. (rhodiola, golden root), which is traditionally used to improve physical and mental performance and endurance. ${ }^{25,26}$ Various extracts of rhodiola have been shown to produce interesting antidepressant effects in preclinical and clinical studies. ${ }^{25,27-30}$

On the assumption that supplementation with a combination of these two plants could be of benefit for depressive patients, we conducted an observational study in which a 6-week supplementation with a fixed combination of rhodiola and saffron extracts was evaluated in adults diagnosed with mild-moderate major depressive disorder (MDD). The study was performed in general practices, since depression is highly prevalent in primary care. ${ }^{2}$

\section{Methods}

\section{Study design and ethics statement}

This was an open-label, observational, longitudinal study performed in France between November 2016 and March 2017. The study was conducted in a context of routine practice: all measures were undertaken and supplement used in a usual way without any additional or unusual procedure of diagnosis or surveillance. The study was approved by the relevant French authorities for this type of study: the advisory committee on information processing in health research (Comite Consultatif sur le Traitement de l'Information en Matière de Recherche dans le Domaine de la Santé [CCTIRS]) and the National Commission on Informatics and Liberty. The study was performed in accordance with the ethical standards laid down in the Declaration of Helsinki and STROBE (strengthening the reporting of observational studies in epidemiology) guidelines. All patients received written information about the study and gave oral consent (approved by CCTIRS) before participation. The study was recorded on ClinicalTrials.gov site December 2016 (NCT02981225).

\section{Participants and recruitment}

Adult patients aged 18-85 years of either sex were recruited by general practitioners (GPs) used to recommending dietary supplement to patients suffering from mild and moderate depression: patients would have received the supplement regardless of whether or not the study were taking place. Patients had 1) to suffer from mild or moderate MDD according to the ICD $10^{31}$ and to have a Hamilton Rating Scale for Depression (HRSD) score of 8-18 (17 items). ${ }^{32}$ Exclusion criteria were patients using an antidepressant or having discontinued it less than a month ago, severe MDD defined by the ICD10 or HRSD score $>18$, having attempted suicide or suicidal (based on the GP's opinion or HRSD item $3>2$ ), psychiatric disorders, such as schizophrenia, bipolarity, and addiction, using chronic treatments (arterial hypertension, cardiac or renal insufficiency, for example), using medications containing piperine or St John's Wort (risk of interaction), and pregnant or lactating women.

\section{Supplementation}

The dietary supplement was a combination of rhodiola and saffron extracts (Phytostandard de Rhodiole et Safran; PiLeJe Laboratoire, Paris, France) marketed in France since 2015. One tablet contains $154 \mathrm{mg}$ of an extract of rhodiola ( $R$. rosea L., roots) and $15 \mathrm{mg}$ of an extract of saffron (C. sativus L., stigmas). The supplement was recommended to be taken at the dose of two tablets per day for 6 weeks.

\section{Procedure}

The procedure was compliant with routine practice and the official recommendations for the management of depression. ${ }^{33}$ On the first visit (V1, day 0 [D0]), eligibility of patients was 
checked. GPs completed an electronic inclusion form with sociodemographic and anthropometric characteristics (age, sex, height, and weight), medical and depression history, information on depression, associated symptoms, concomitant pathologies, and treatments. GPs assessed patients' depression status with the HRSD. The original version contains 17 items. The total score is interpreted as normal (0-7), mild (8-13), moderate (14-18), severe (19-22), and very severe depression $(>23)$. Patients' clinical condition was assessed by the GP with the Clinical Global Impressionseverity (CGI-S) scale evaluating the severity of psychopathology on D1-D7. The day after V1, patients completed an electronic notebook with information on their depression and completed the Hospital Anxiety and Depression Scale (HADS). The HADS, commonly used to determine levels of anxiety and depression, is a 14-item scale: seven items relate to anxiety and seven to depression. The HADS was also completed after 2, 4, and 6 weeks of supplementation. The Patient Global Impression of Change (PGIC) scale, a 9-point self-rated questionnaire assessing the change of patient clinical condition following a treatment, was completed by patients after 2, 4, and 6 weeks of supplementation. On the second visit after 6 weeks of supplementation (V2, D42), GPs performed a medical examination to reassess the situation. Adverse events and concomitant therapies were collected in the source medical record and on an electronic end-of-visit form. Patient depression status according to the HRSD was reassessed, as well as the CGI-S. CGI - improvement (CGI-I) was also used by the GP to evaluate the change from the initiation of treatment on a 7-point scale. Satisfaction and compliance were evaluated.

\section{Evaluation criteria}

The main end point was the evolution of HRSD score (components 1-17) after 6 weeks of supplementation. HADS, PGIC, CGI-S, and CGI-I scores, patient satisfaction, safety, and compliance were secondary-evaluation criteria.

\section{Statistical analysis}

According to results observed in several published studies with saffron and rhodiola extracts, a 4-point decrease in HRSD score after 6 weeks of supplementation was deemed relevant. ${ }^{17,23,24,30}$ Given a 4-point decrease in HRSD score and considering a risk of error of $\alpha=5 \%$, SD of 5 points, intrapatient correlation of 0.1 , and power of $90 \%$, the sample size was estimated to be at least 26 patients (paired $t$-test, one-sided).

Continuous variables are presented as mean \pm SD. Categorical variables are presented as percentages. Paired
Student's $t$-tests or Wilcoxon signed-rank tests were used depending on the normality or abnormality of data distribution for the comparison of HRSD, HADS, and CGI scores. A Spearman correlation test was performed to analyze the correlation between the evolution of HRSD score and its value at baseline. Analyses were performed on intention-totreat (ITT), modified ITT (mITT; patients who took at least one dose of supplementation and who had a V2), and per protocol (PP) populations. Safety analysis was performed on all patients who had received at least one dose of treatment. All statistical analyses were performed with SAS software version 9.4. In all tests, $P<0.05$ was considered statistically significant.

\section{Results}

A total of 59 patients were screened and enrolled by 16 GPs between November 2016 and March 2017. Five patients were lost to follow-up (including the loss of two patients due to discontinuation of one GP) and nine were excluded for not meeting inclusion criteria (Figure 1). Therefore, 45 patients who had received at least one dose of supplement were included in the safety population. During the supplementation period, four patients dropped out (three mistakenly included and one lost to follow-up). In the end, 41 patients completed the study and were included in the mITT population. Four were excluded from the PP population because of poor compliance or intake of concomitant medications that were not authorized. As such, 37 patients were included in the PP population.

\section{Patient characteristics}

Patients were mainly women $(82.2 \%, 37$ of 45 patients, safety population; Table 1), among which $43.2 \%$ were menopausal (16 of 37). Mean age at inclusion was $47.6 \pm 14.6$ years. A total of 28 of 45 patients $(63.6 \%)$ had declared in their electronic notebooks suffering from depression at least once in their life; five of $28(17.9 \%)$ had taken antidepressants. The first depressive episode had occurred $13 \pm 12$ years $(156.2 \pm 144.7$ months) before inclusion (median 10.5 [0.5-50.0] years). Seven of 45 patients $(15.6 \%)$ had family members who had a history of depression, more frequently the mother (four of seven, $57.1 \%$ ). The average duration of the ongoing depressive episode was $4.4 \pm 4.5$ (median 3 [1-24]) months.

Eighteen of 45 patients (40\%) had had surgery or a medical history other than depression. Fourteen of 45 patients (31.1\%) continued their ongoing treatment (other than for depression) during supplementation, with a total of 28 treatments declared. The drugs were taken more frequently for 




Figure I Flow diagram.

Abbreviations: GP, general practitioner; mITT, modified intention-to-treat; $\mathrm{D} 42$, day 42; PP, per protocol.

obstructive airway diseases $(20 \%$, nine of 45$)$, cardiovascular diseases (15.6\%, seven of 45$)$, and nervous-system disorders (11.1\%, five of 45$)$.

\section{HRSD scores}

A significant decrease in HRSD scores of $58 \% \pm 28.5 \%$ was observed after 6 weeks of supplementation: from $13.6 \pm 2.3$ at

Table I Patient characteristics

\begin{tabular}{lll}
\hline & $\begin{array}{l}\text { Safety } \\
\text { population } \\
(\mathbf{n}=\mathbf{4 5})\end{array}$ & $\begin{array}{l}\text { PP } \\
\text { population } \\
(\mathbf{n}=\mathbf{3 7})\end{array}$ \\
\hline Women & $37(82.2 \%)$ & $30(81.1 \%)$ \\
Men & $8(17.8 \%)$ & $7(18.9 \%)$ \\
Age (years) & $47.6 \pm 14.6$ & $47.0 \pm 14.2$ \\
Patient history of depression & $20(44.4 \%)$ & $16(43.2 \%)$ \\
Family history of depression & $7(15.6 \%)$ & $7(18.9 \%)$ \\
Duration of ongoing & $4.4 \pm 4.5$ & $4.0 \pm 3.6$ \\
depressive episode (months) & $18(40.0 \%)$ & $15(40.5 \%)$ \\
Surgery or medical history
\end{tabular}

Note: apatients who declared having suffered or suffering from at least one disease other than depression.

Abbreviation: PP, per protocol.
D0 to $5.6 \pm 3.8$ at $\mathrm{D} 42(7.9 \pm 4.2,95 \%$ CI $9.29-6.61 ; P<0.0001$, $\mathrm{n}=41)$. At D42, HRSD score had reduced by at least $20 \%$ in $87.8 \%$ of patients ( 36 of $41,95 \%$ CI $74.00 \%-95.14 \%$ ). According to HRSD-score classes, $53.7 \%$ (22 of 41) of patients had mild depression and 46.3\% (19 of 41) moderate depression at D0. After 6 weeks of supplementation, HRSD score had decreased by $53.7 \% \pm 29.6 \%$ (6.3 \pm 3.6 points) in mildly depressive patients $(\mathrm{n}=22)$, whereas it had decreased by $62.9 \% \pm 27 \%(9.8 \pm 4.3$ points $)$ in patients suffering from moderate depression $(n=19)$. The Spearman correlation coefficient between HRSD-score evolution and HRSD score at inclusion was -0.5 , indicating significant opposing variation trends between the two variables: score decreases were greatest in patients with the highest scores at D0.

At D42, depressive symptoms had improved in $85.4 \%$ of patients (35 of 41 ), were stable in $12.2 \%$ (five of 41 ), and worse in one patient $(2.4 \%)$. Patient distribution into the HRSD classes was significantly different from that observed at D0 $(P<0.0001$, Figure 2$)$, with 29 patients $(70.7 \%, 29$ of 41$)$ 


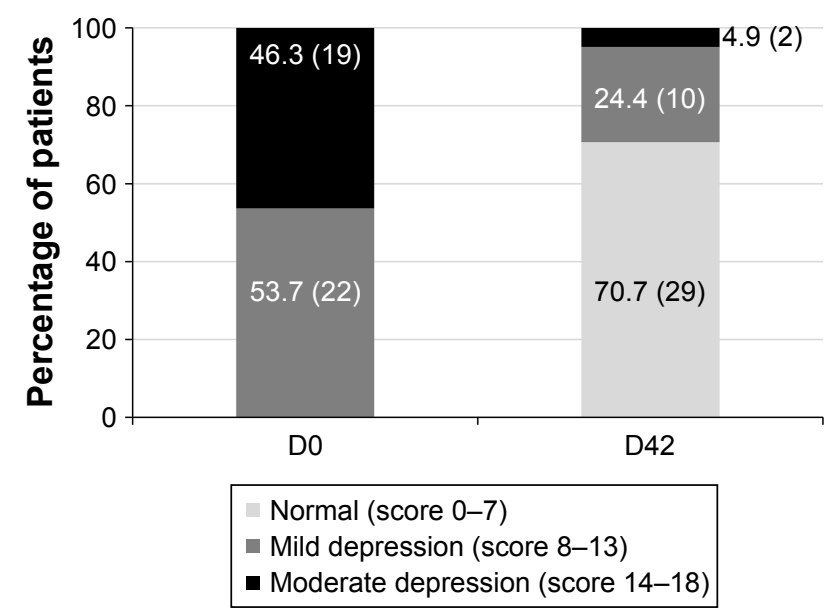

Figure 2 Patients' distribution into HRSD classes at day 0 (D0) and D42 (\% [n], modified intention-to-treat population)

Abbreviation: HRSD, Hamilton Rating Scale for Depression.

no longer suffering from depression at D42. In addition, only two of 41 patients $(4.9 \%)$ were suffering from moderate depression at D42 compared to 19 of $41(46.3 \%)$ at D0.

\section{HADS scores}

A significant drop in both the HADS anxiety and depression scores was observed between D0 and D42. The decrease was $31.3 \% \pm 23.2 \%$ for HADS anxiety: from $12.0 \pm 3.1$ at D0 to $8.0 \pm 3.2$ at $\mathrm{D} 42(3.9 \pm 3.3,95 \%$ CI $2.86-4.99 ; P<0.0001)$, with a decrease of $46.1 \% \pm 48.2 \%$ for HADS depression from $10.0 \pm 3.6$ at $\mathrm{D} 0$ to $5.5 \pm 4.8$ at $\mathrm{D} 42(4.4 \pm 5.5,95 \%$ CI $3.09-6.40$; $P<0.0001)$. The drop was significant after 2 weeks of supplementation and throughout follow-up (Figure 3).

HADS anxiety scores decreased by at least $20 \%$ in $71.8 \%$ of patients ( 28 of $39,95 \%$ CI $56.10 \%-83.58 \%$ ) and HADS depression scores decreased by at least $20 \%$ in $66.7 \%$ (26 of $39,95 \%$ CI $50.90 \%-79.44 \%$ ). After 6 weeks of supplementation, patient distribution into the HADS classes was significantly different from that observed at D0 for the two scores $(P<0.0001$ for HADS anxiety and $P=0.0005$ for HADS depression, Figure 4). Globally, there was a progressive decline in prevalence of anxiety and depressive symptoms throughout the study.

\section{CGI and PGIC scores}

According to GPs' answers to CGI-S, only 14.6\% (6 of 41) of patients were moderately or markedly depressive after 6 weeks of supplementation compared to $75.6 \%$ (31 of 41) before supplementation (Wilcoxon test $P<0.0001$, Table 2). GPs deemed that depression was very much improved in $78.1 \%$ (32 of 41) of patients after supplementation (CGI-I). In the PGIC, $56.4 \%$ (22 of 41) of patients declared feeling better after 2 weeks of supplementation, $62.5 \%$ (25 of 41 ) after 4 weeks, and $74.4 \%$ (29 of 41) after 6 weeks. All significant results in the mITT population were also significant in the PP population.

\section{Compliance and safety}

In the mITT population, information on compliance was available for 39 patients of 41 . Thirty $(76.9 \%)$ declared taking the supplementation without interruption and nine $(23.1 \%)$ with a few missed days. Six patients of 45 in the safety population experimented at least one adverse event. Nine adverse events were listed: one dizziness, one vision blurred, one arthralgia, one lower-abdomen pain, one dry mouth, one fatigue, one nausea, and two upper-abdomen pain. These adverse events were of mild severity, completely recovered before the end of supplementation, and did not lead to discontinuation of supplementation. No serious adverse events were reported.

\section{Discussion}

In this preliminary study performed with recognized and validated criteria and measurement scales for the diagnosis
A

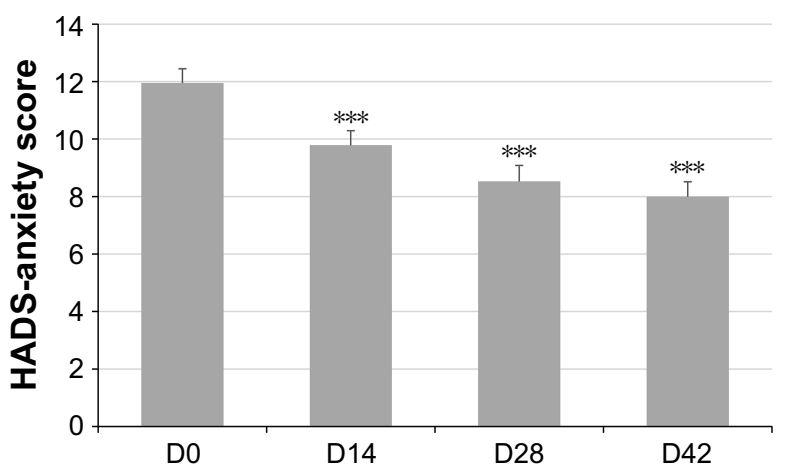

B

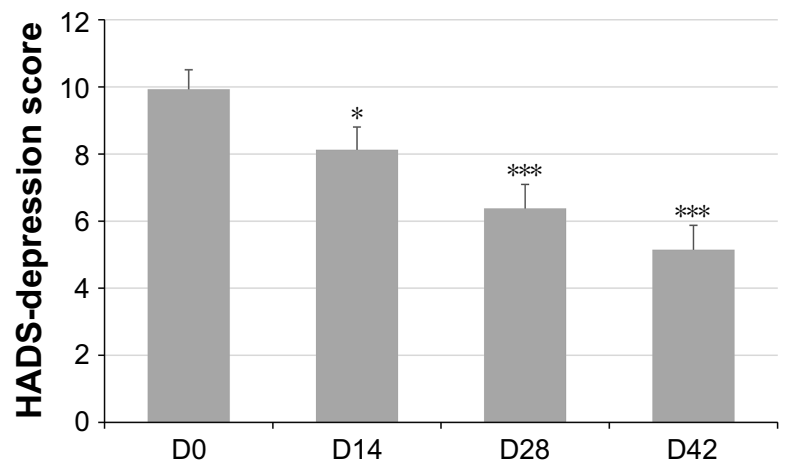

Figure 3 Evolution of HADS anxiety (A) and HADS depression (B) scores between day 0 (D0; $n=40, m$ ITT population) and D42 ( $n=39$ ).

Notes: Data shown as mean \pm SEM. $* P<0.05, * * * P<0.000$ I versus D0.

Abbreviations: HADS, Hospital Anxiety and Depression Scale; mITT, modified intention-to-treat. 


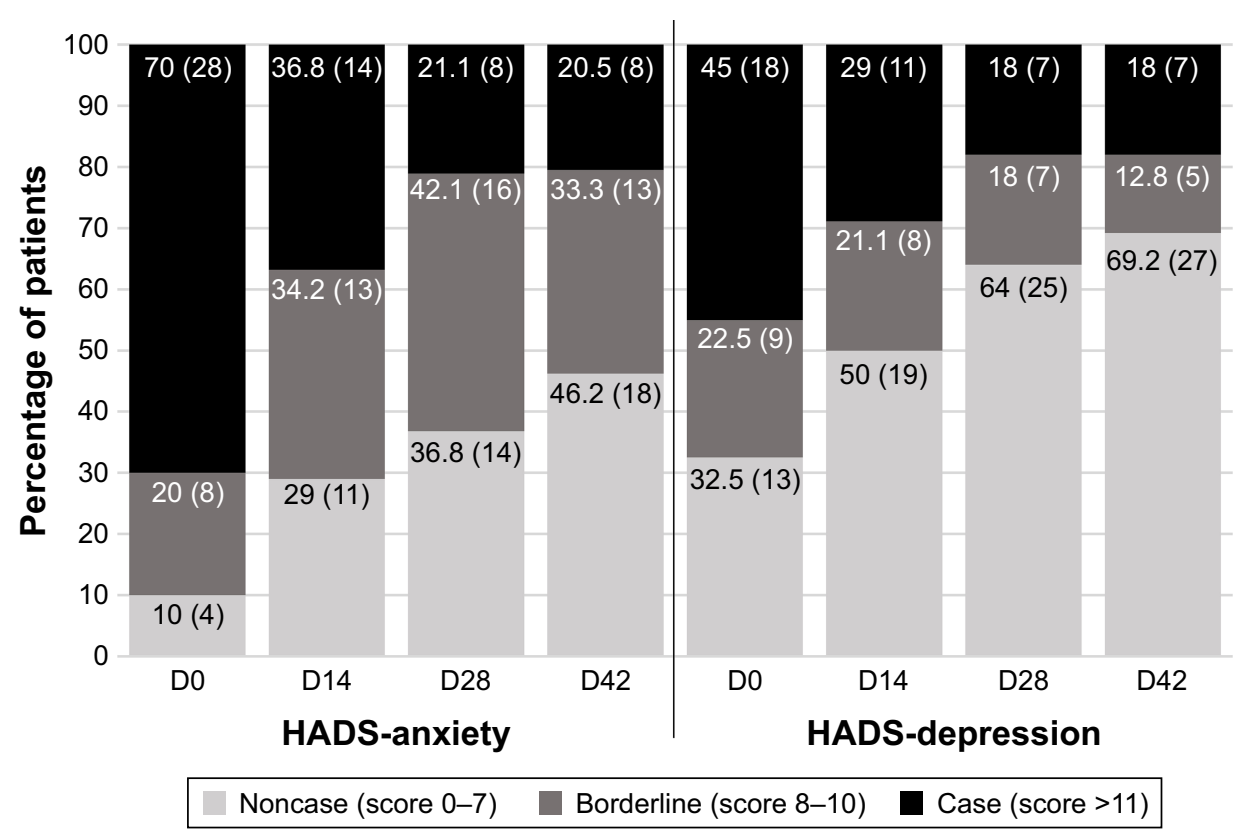

Figure 4 Evolution of patients' distribution into HADS classes between day 0 (D0) and D42 (\% [n]; modified intention-to-treat population). Abbreviation: HADS, Hospital Anxiety and Depression Scale.

and assessment of depression and anxiety, 6-week supplementation with a combination of rhodiola and saffron extracts was associated with a significant decrease in severity of depression in patients diagnosed as mildly to moderately ill at inclusion. Mean HRSD scores decreased by $\sim 58 \%$ ( $~ 8$ points) between the first and last visit 6 weeks later. To our knowledge, this is the first time that such a combination of rhodiola and saffron has been tested. Previously, rhodiola and saffron extracts were shown to have beneficial effects in patients suffering from MDD, but separately. 17,18,21-24,26,30
The efficacy of saffron for the treatment of mild-moderate depression has been shown in several randomized doubleblind clinical trials. In these studies, an extract of $C$. sativus stigmas at a dose of $30 \mathrm{mg}$ per day for 6 weeks ${ }^{24}$ and $100 \mathrm{mg}$ per day for 12 weeks ${ }^{21}$ was more effective than placebo. This extract ( $30 \mathrm{mg} /$ day for 6 weeks) had similar effects to those of fluoxetine $(20 \mathrm{mg} / \text { day })^{17}$ and imipramine $(100 \mathrm{mg} /$ day $){ }^{23}$ Comparable effects were reported with a petal extract of saffron at $30 \mathrm{mg}$ per day for 6 weeks vs placebo ${ }^{18}$ and for 8 weeks vs fluoxetine $(20 \mathrm{mg} /$ day $) .{ }^{19}$

Table $2 \mathrm{CGI}$ and PGIC scores in the mITT population

\begin{tabular}{|c|c|c|c|c|}
\hline CGI-S & Do & D42 & CGI-I & D42 \\
\hline Normal, not at all ill & $2.4 \%(I / 4 I)$ & $5 \mathrm{I} .2 \%(2 \mathrm{I} / 4 \mathrm{I})$ & Very much improved & $56.1 \%(23 / 4 \mid)$ \\
\hline Borderline & $4.9 \%(2 / 4 I)$ & $21.9 \%(9 / 4 I)$ & Much improved & $21.9 \%(9 / 4 I)$ \\
\hline Mildly ill & $17.1 \%(7 / 4 \mid)$ & $12.2 \%(5 / 4 \mid)$ & Slightly improved & $12.2 \%(5 / 4 \mid)$ \\
\hline Moderately ill & $39.0 \%(|6 / 4|)$ & $12.2 \%(5 / 4 \mid)$ & No change & $7.3 \%(3 / 4 I)$ \\
\hline Markedly ill & $36.6 \%(15 / 4 I)$ & $2.4 \%(I / 4 I)$ & Minimally worse & $2.4 \%(I / 4 I)$ \\
\hline \multicolumn{2}{|l|}{ PGIC } & DI4 & D28 & D42 \\
\hline \multicolumn{2}{|l|}{ Worsening } & $2.6 \%(I / 39)$ & 0 & 0 \\
\hline \multicolumn{2}{|l|}{ No change } & $2.6 \%(I / 39)$ & $12.5 \%(5 / 40)$ & $7.7 \%(3 / 39)$ \\
\hline \multicolumn{2}{|c|}{ Almost the same, hardly any change } & $12.8 \%(5 / 39)$ & $7.5 \%(3 / 40)$ & $10.3 \%(4 / 39)$ \\
\hline \multicolumn{2}{|c|}{ A little bit better, but no noticeable change } & $20.5 \%(8 / 39)$ & $10 \%(4 / 40)$ & $5.1 \%(2 / 39)$ \\
\hline \multicolumn{2}{|c|}{ Somewhat better, but change does not make any real difference } & $5.1 \%(2 / 39)$ & $7.5 \%(3 / 40)$ & $2.6 \%(1 / 39)$ \\
\hline \multicolumn{2}{|c|}{ Better, moderate change, but noticeable } & $41 \%(16 / 39)$ & $12.5 \%(5 / 40)$ & $20.5 \%(8 / 39)$ \\
\hline \multicolumn{2}{|c|}{ Better, with a definite improvement that makes the difference } & $12.8 \%(5 / 39)$ & $35 \%(14 / 40)$ & $15.4 \%(6 / 39)$ \\
\hline \multicolumn{2}{|c|}{$\begin{array}{l}\text { A great deal better, and a considerable improvement that } \\
\text { makes all the difference }\end{array}$} & $2.6 \%(1 / 39)$ & $12.5 \%(5 / 40)$ & $33.3 \%(13 / 39)$ \\
\hline \multicolumn{2}{|l|}{ Complete recovery } & 0 & $2.5 \%(1 / 40)$ & $5.1 \%(2 / 39)$ \\
\hline
\end{tabular}

Abbreviations: CGI-S, Clinical Global Impression - severity; PGIC, Patient Global Impression of Change; mITT, modified intention to treat. 
In a randomized double-blind placebo-controlled clinical trial performed in 89 adults with mild-moderate DSM-IV MDD, Beck Depression Inventory and HRSD scores significantly declined in patients having received 340 or $680 \mathrm{mg}$ of $R$. rosea rhizome extract (SHR5) per day over a 6-week period in comparison with patients having received the placebo, for whom scores were unchanged..$^{30}$ Overall depression, along with insomnia, emotional instability, somatization (at both doses), and self-esteem (only at the highest dose) were significantly improved after supplementation with $R$. rosea. In another randomized double-blind clinical trial in mildmoderate depression (57 patients randomized), supplementation with the same extract of $R$. rosea, also administered at $340 \mathrm{mg}$ per day, but this time for 12 weeks, was compared to sertraline $(50 \mathrm{mg} /$ day $)$ and placebo. ${ }^{34}$ There was no significant difference between groups in changes in HRSD, Beck Depression Inventory, or CGI scores over time.

$R$. rosea is more known for its adaptogenic properties than antidepressant effects, and has proven benefits in patients with stress-related fatigue and anxiety. ${ }^{35-37}$ An anxiolytic effect has also been reported for saffron. ${ }^{22,38}$ In our study, after 6 weeks of supplementation with a combination of rhodiola and saffron, HADS anxiety scores were reduced significantly by $>30 \%$. This anxiolytic effect is particularly interesting, because the concurrent presence of a depressive disorder with prominent anxiety symptoms or an anxiety disorder is common in clinical practice. ${ }^{39-41}$ It was estimated that $>70 \%$ of individuals with depressive disorders also have anxiety symptoms and $40 \%-70 \%$ simultaneously met criteria for at least one type of anxiety disorder. ${ }^{39,41}$ Previous studies have shown that patients with MDD with high levels of anxiety have a slower response to treatment, ${ }^{42}$ and in some ${ }^{41,43-46}$ but not all studies ${ }^{47,48}$ were less likely to respond to antidepressants than patients with low levels of anxiety. In depressive patients treated with common antidepressants, such as citalopram and sertraline, more severe anxiety symptoms before treatment were associated with lower remission rates across all medications. ${ }^{46}$ In some guidelines, it is considered that effective treatment of anxiety disorders will improve depression. ${ }^{12}$

In addition to the evaluation of depression and anxiety symptoms, both GPs and the patients were given the opportunity to rate their impression of the supplementation and its effects using the CGI and PGIC scales. The CGI allows clinicians to assess clinically relevant global response to treatment. ${ }^{49}$ It has become one of the most widely used assessment tools in psychiatry, especially as an efficacy measure in clinical drug trials in different mental disorders, ${ }^{50}$ as it makes results applicable for clinical practice. In our study, according to CGI-I scores, depression was much and very much improved in $\sim 80 \%$ of patients, which was confirmed by the significant decrease in severity (CGI-S score). As an example, this scale has been used in a randomized doubleblind placebo-controlled clinical trial in which 207 patients were treated with either a Hypericum extract or citalopram for a 6 -week period. At the end of the study, according to the CGI-I, $\sim 65 \%$ of patients treated with Hypericum and $\sim 68 \%$ of patients treated with citalopram had their depression rated as much and very much improved. ${ }^{51}$

The PGIC is a clinical tool built to assess the patient's perceived impact of disease management. It allows patients to rate their overall status from the start of a study. ${ }^{52}$ The scale is based on function and symptoms that are important for each individual patient. It provides a responsive and readily interpretable measure of participant assessment of the clinical importance of their improvement or worsening over the course of a study. In our study, nearly $75 \%$ of patients declared feeling better after supplementation, suggesting a beneficial impact of the combination of rhodiola and saffron on patients' perception of their disorder.

The main limitation of our study is obviously the absence of a control group. This study was observational; therefore, the effect of the combination tested might have been overestimated. The combination tested was well tolerated, with nine adverse events reported by six patients. These adverse events were of mild severity and did not lead to discontinuation of supplementation. Minor side effects have been associated with rhodiola, including dizziness and dry mouth. ${ }^{53} \mathrm{~A}$ root extract of rhodiola was previously reported to be associated with fewer adverse events and better tolerated than a conventional antidepressant in patients with depression. ${ }^{34}$ No adverse events were observed in Darbinyan et al. ${ }^{30}$ In placebo- and antidepressant-controlled clinical trials, saffron was mainly associated with nausea, decreased appetite, anxiety, and headache, but those side effects were also observed with placebo and much less numerous than with antidepressants. ${ }^{22,54}$

\section{Conclusion}

Altogether, the results of this preliminary study performed in primary care suggest that the tested supplement containing rhodiola and saffron extracts could be of interest for the management of mild-moderate depression. There was a rapid improvement in both depressive and anxiety symptoms as assessed with the HRSD and the HADS, the CGI was much improved, and according to the PGIC a majority of patients felt better after supplementation. In addition, the combination of rhodiola and saffron was well tolerated. Benefits of the 
combination will have to be confirmed in a double-blind placebo-controlled study.

\section{Disclosure}

MB, SAA, AB, CB, AG, and MD are employees of Groupe PiLeJe. PL is a consultant for PiLeJe. The authors report no other conflicts of interest in this work.

\section{References}

1. Hirschfeld RM. The epidemiology of depression and the evolution of treatment. J Clin Psychiatry. 2012;73(Suppl 1):5-9.

2. Craven MA, Bland R. Depression in primary care: current and future challenges. Can J Psychiatry. 2013;58(8):442-448.

3. World Health Organization. Depression and Other Common Mental Disorders: Global Health Estimates. Geneva: WHO; 2017.

4. World Health Organization. Dépression. 2018. Available from: http:// www.who.int/mediacentre/factsheets/fs369/fr. Accessed February 28, 2018.

5. Wittchen HU, Mühlig S, Beesdo K. Mental disorders in primary care. Dialogues Clin Neurosci. 2003;5(2):115-128.

6. Demyttenaere K. Compliance during treatment with antidepressants. J Affect Disord. 1997;43(1):27-39.

7. Rush AJ, Trivedi MH, Wisniewski SR, et al. Acute and longer-term outcomes in depressed outpatients requiring one or several treatment steps: a STAR*D report. Am J Psychiatry. 2006;163(11):1905-1917.

8. Fournier JC, Derubeis RJ, Hollon SD, et al. Antidepressant drug effects and depression severity: a patient-level meta-analysis. JAMA. 2010;303(1):47-53.

9. Zimmermann TM, Clouth J, Elosge M, et al. Patient preferences for outcomes of depression treatment in Germany: a choice-based conjoint analysis study. J Affect Disord. 2013;148(2-3):210-219.

10. World Health Organization. Antidepressants (tricyclic antidepressants and selective serotonin reuptake inhibitors) in treatment of adults with depression. 2010. Available from: http://www.who.int/mental_health/ mhgap/evidence/depression/mh_evidence_profile_q1_tca_ssri_2010_ en.pdf. Accessed February 27, 2018.

11. Haute Autorité de Santé. Episode dépressif caractérisé de l'adulte: prise en charge en soins de premier recours. 2017. Available from: https:// www.has-sante.fr/portail/upload/docs/application/pdf/2017-10/depression_adulte_recommandations_version_mel.pdf. Accessed March 27, 2018.

12. National Institute for Health and Care Excellence. Depression in Adults: Recognition and Management. London: NICE; 2009.

13. Mitchell J, Trangle M, Degnan B, et al. Adult depression in primary care. 2013. Available from: http://pcptoolkit.beaconhealthoptions. com/wp-content/uploads/2016/02/ICSI_Depression.pdf. Accessed March 27, 2018.

14. Michigan Quality Improvement Consortium. Primary care diagnosis and management of adults with depression. 2018. Available from: http://www.mqic.org/pdf/mqic_primary_care_diagnosis_and_management_of_adults_with_depression_cpg.pdf. Accessed March 1, 2018.

15. Anthes E. Depression: a change of mind. Nature. 2014;515(7526): $185-187$.

16. Warrilow AE, Beech B. Self-help CBT for depression: opportunities for primary care mental health nurses? J Psychiatr Ment Health Nurs. 2009;16(9):792-803.

17. Noorbala AA, Akhondzadeh S, Tahmacebi-Pour N, Jamshidi AH. Hydro-alcoholic extract of Crocus sativus L. versus fluoxetine in the treatment of mild to moderate depression: a double-blind, randomized pilot trial. J Ethnopharmacol. 2005;97(2):281-284.

18. Moshiri E, Basti AA, Noorbala AA, Jamshidi AH, Abbasi SH, Akhondzadeh S. Crocus sativus L. (petal) in the treatment of mildto-moderate depression: a double-blind, randomized and placebocontrolled trial. Phytomedicine. 2006;13(9-10):607-611.
19. Basti AA, Moshiri E, Noorbala AA, Jamshidi AH, Abbasi SH, Akhondzadeh S. Comparison of petal of Crocus sativus L. and fluoxetine in the treatment of depressed outpatients: a pilot double-blind randomized trial. Prog Neuropsychopharmacol Biol Psychiatry. 2007; 31(2):439-442.

20. Hausenblas HA, Saha D, Dubyak PJ, Anton SD. Saffron (Crocus sativus $\mathrm{L}$.) and major depressive disorder: a meta-analysis of randomized clinical trials. J Integr Med. 2013;11(6):377-383.

21. Mazidi M, Shemshian M, Mousavi SH, et al. A double-blind, randomized and placebo-controlled trial of saffron (Crocus sativus L.) in the treatment of anxiety and depression. J Complement Integr Med. 2016;13(2): 195-199.

22. Shafiee M, Arekhi S, Omranzadeh A, Sahebkar A. Saffron in the treatment of depression, anxiety and other mental disorders: current evidence and potential mechanisms of action. J Affect Disord. 2018;227: 330-337.

23. Akhondzadeh S, Fallah-Pour H, Afkham K, Jamshidi AH, KhalighiCigaroudi F. Comparison of Crocus sativus L. and imipramine in the treatment of mild to moderate depression: a pilot double-blind randomized trial [ISRCTN45683816]. BMC Complement Altern Med. 2004; $4: 12$.

24. Akhondzadeh S, Tahmacebi-Pour N, Noorbala AA, et al. Crocus sativus L. in the treatment of mild to moderate depression: a double-blind, randomized and placebo-controlled trial. Phytother Res. 2005;19(2): 148-151.

25. Amsterdam JD, Panossian AG. Rhodiola rosea L. as a putative botanical antidepressant. Phytomedicine. 2016;23(7):770-783.

26. Yeung KS, Hernandez M, Mao JJ, Haviland I, Gubili J. Herbal medicine for depression and anxiety: a systematic review with assessment of potential psycho-oncologic relevance. Phytother Res. 2018;32(5): 865-891.

27. Brichenko V, Kupriyanova I, Skorokhodova T. The use of herbal adaptogens together with tricyclic antidepressants in patients with psychogenic depressions. In: Goldberg ED, editor. Modern Problems of Pharmacology and Search for New Medicines. Tomsk: Tomsk University Press; 1986:58-60.

28. Brichenko V, Skorokhodova T. Herbal Adaptogens in Rehabilitation of Patients with Depression: Clinical and Organisational Aspects of Early Manifestations of Nervous and Mental Diseases. Vol 15. Barnaul: Altajskoe Knižnoe; 1987.

29. Perfumi M, Mattioli L. Adaptogenic and central nervous system effects of single doses of $3 \%$ rosavin and $1 \%$ salidroside Rhodiola rosea $\mathrm{L}$. extract in mice. Phytother Res. 2007;21(1):37-43.

30. Darbinyan V, Aslanyan G, Amroyan E, Gabrielyan E, Malmström C, Panossian A. Clinical trial of Rhodiola rosea L. extract SHR-5 in the treatment of mild to moderate depression. Nord J Psychiatry. 2007; 61(5):343-348.

31. World Health Organization. Mental and behavioural disorders. In: International Statistical Classification of Diseases and Related Health Problems. 10th ed. Geneva: WHO; 2016:F00-F99.

32. Hamilton M. A rating scale for depression. J Neurol Neurosurg Psychiatry. 1960;23(1):56-62.

33. Maj M. Development and validation of the current concept of major depression. Psychopathology. 2012;45(3):135-146.

34. Mao JJ, Xie SX, Zee J, et al. Rhodiola rosea versus sertraline for major depressive disorder: a randomized placebo-controlled trial. Phytomedicine. 2015;22(3):394-399.

35. Cropley M, Banks AP, Boyle J. The effects of Rhodiola rosea L. extract on anxiety, stress, cognition and other mood symptoms. Phytother Res. 2015;29(12):1934-1939.

36. Olsson EM, von Schéele B, Panossian AG. A randomised, double-blind, placebo-controlled, parallel-group study of the standardised extract SHR-5 of the roots of Rhodiola rosea in the treatment of subjects with stress-related fatigue. Planta Med. 2009;75(2):105-112.

37. Anghelescu IG, Edwards D, Seifritz E, Kasper S. Stress management and the role of Rhodiola rosea: a review. Int J Psychiatry Clin Pract. Epub 2018 Jan 11. 
38. Basiri-Moghadam M, Hamzei A, Moslem AR, Pasban-Noghabi S, Ghorbani N, Ghenaati J. Comparison of the anxiolytic effects of saffron (Crocus sativus. L) and diazepam before herniorrhaphy surgery: a double blind randomized clinical trial. Zahedan J Res Med Sci. 2016; 18(3):e6248.

39. Wu Z, Fang Y. Comorbidity of depressive and anxiety disorders: challenges in diagnosis and assessment. Shanghai Arch Psychiatry. 2014;26(4):227-231.

40. Lamers F, van Oppen P, Comijs HC, et al. Comorbidity patterns of anxiety and depressive disorders in a large cohort study: the Netherlands Study of Depression and Anxiety (NESDA). J Clin Psychiatry. 2011; 72(3):341-348.

41. Fava M, Rush AJ, Alpert JE, et al. Difference in treatment outcome in outpatients with anxious versus nonanxious depression: a STAR*D report. Am J Psychiatry. 2008;165(3):342-351.

42. Clayton PJ, Grove WM, Coryell W, Keller M, Hirschfeld R, Fawcett J. Follow-up and family study of anxious depression. Am J Psychiatry. 1991;148(11):1512-1517.

43. Fava M, Uebelacker LA, Alpert JE, Nierenberg AA, Pava JA, Rosenbaum JF. Major depressive subtypes and treatment response. Biol Psychiatry. 1997;42(7):568-576.

44. Flint AJ, Rifat SL. Anxious depression in elderly patients: response to antidepressant treatment. Am J Geriatr Psychiatry. 1997;5(2): 107-115.

45. Davidson JR, Meoni P, Haudiquet V, Cantillon M, Hackett D. Achieving remission with venlafaxine and fluoxetine in major depression: its relationship to anxiety symptoms. Depress Anxiety. 2002;16(1):4-13.

46. Saveanu R, Etkin A, Duchemin AM, et al. The International Study to Predict Optimized Treatment in Depression (iSPOT-D): outcomes from the acute phase of antidepressant treatment. J Psychiatr Res. 2015;61: $1-12$.
47. Tollefson GD, Holman SL, Sayler ME, Potvin JH. Fluoxetine, placebo, and tricyclic antidepressants in major depression with and without anxious features. J Clin Psychiatry. 1994;55(2):50-59.

48. Russell JM, Koran LM, Rush J, et al. Effect of concurrent anxiety on response to sertraline and imipramine in patients with chronic depression. Depress Anxiety. 2001;13(1):18-27.

49. Leucht S, Fennema H, Engel R, Kaspers-Janssen M, Lepping P, Szegedi A. What does the HAMD mean? J Affect Disord. 2013; 148(2-3):243-248.

50. Forkmann T, Scherer A, Boecker M, Pawelzik M, Jostes R, Gauggel S. The Clinical Global Impression scale and the influence of patient or staff perspective on outcome. BMC Psychiatry. 2011;11:83.

51. Gastpar M, Singer A, Zeller K. Comparative efficacy and safety of a once-daily dosage of Hypericum extract STW3-VI and citalopram in patients with moderate depression: a double-blind, randomised, multicentre, placebo-controlled study. Pharmacopsychiatry. 2006;39(2): 66-75.

52. Rampakakis E, Ste-Marie PA, Sampalis JS, Karellis A, Shir Y, Fitzcharles MA. Real-life assessment of the validity of patient global impression of change in fibromyalgia. RMD Open. 2015;1(1): e000146.

53. Bystritsky A, Kerwin L, Feusner JD. A pilot study of Rhodiola rosea (Rhodax) for generalized anxiety disorder (GAD). J Altern Complement Med. 2008;14(2):175-180.

54. Lopresti AL, Drummond PD. Saffron (Crocus sativus) for depression: a systematic review of clinical studies and examination of underlying antidepressant mechanisms of action. Hum Psychopharmacol. 2014; 29(6):517-527.
Neuropsychiatric Disease and Treatment

\section{Publish your work in this journal}

Neuropsychiatric Disease and Treatment is an international, peerreviewed journal of clinical therapeutics and pharmacology focusing on concise rapid reporting of clinical or pre-clinical studies on a range of neuropsychiatric and neurological disorders. This journal is indexed on PubMed Central, the 'PsycINFO' database and CAS,

\section{Dovepress}

and is the official journal of The International Neuropsychiatric Association (INA). The manuscript management system is completely online and includes a very quick and fair peer-review system, which is all easy to use. Visit http://www.dovepress.com/testimonials.php to read real quotes from published authors. 